\title{
A GENERAL METHOD TO COMPUTE NUMERICAL DISPERSION ERROR
}

\author{
J. RUANO ${ }^{1}$, A. BAEZ VIDAL ${ }^{1}$ J. RIGOLA $^{1}$ AND F.X. TRIAS $^{1}$ \\ ${ }^{1}$ Heat and Mass Transfer Technological Center (CTTC), Universitat Politecnica de Catalunya \\ BarcelonaTech (UPC) ESEIAAT \\ Colom 11, 08222, Terrassa, Barcelona, Spain \\ jesus.ruano@upc.edu, aleix@cttc.upc.edu, joaquim.rigola@upc.edu, francesc.xavier.trias@upc.edu
}

Key words: Dispersion error, Diffusion error, Structured/Unstructured grids, High-order methods

\begin{abstract}
This article presents a new methodology to compute numerical dispersion error. The analysis here presented is not restricted to uniform structured meshes nor linear discrete operators as it does not rely on sinusoids to compute the associated error. When using uniform meshes, the results obtained with the present method collapse onto the obtained with the classic one via an easy change of basis. If non-uniform meshes are used, a new kind of results are obtained which shed some light onto the role stretching has on dispersion error.
\end{abstract}

\section{INTRODUCTION}

Discretization of the different differential operators induces errors. Among others, the study of errors in the advective term in Computational Fluid Dynamics (CFD) or Computational AeroAcoustics (CAA) is still investigated. Diffusion error is totally avoided by preserving the skew-symmetry of the discrete operator, which is only achieved by using central schemes or Symmetry Preserving schemes analysed by Trias et al. [1], Verstapen and Veldman [2] or Rozema et al. [3].

Nevertheless, there are situations where keeping the skew-symmetry of the discrete operation, i.e. having null numerical diffusion, is not the prefered option. Sometimes numerical diffusion is introduced on purpose in order to avoid numerical instabilities. There are a wide number of options, from upwind numerical schemes to Essential Non-Oscillating, Weighted (WENO) [4] or not (ENO) [5], passing from TVD schemes and Flux or Slope Limiters [6].

However, even when the symmetry, or skew-symmetry, of the operator is preserved, there is another kind of unavoidable error: the dispersion error. Only if spectral methods are used, when the derivative is known and not approximated, the dispersion error can be avoided. Tam and Webb [7], Lele [8], Pirozzoli [9] and more recently Fauconnier and Dick [10] have studied the dispersion characteristics of several numerical schemes, ranging from linear ones to completely non-linear, such as previously mentioned WENO or Flux Limiter ones. 
This kind of analysis has concluded that the use of high-order schemes is prefered over loworder ones. For example, in CAA the majority of its researchers use high-order schemes as the correct wave-propagation is the key to obtain acceptable results. In this sense, Bogey [11] and Shur et al. [12, 13] used high-order schemes in their jets simulations. Seo and Moon [14] and Ewert and Schröder [15] solved a different kind of wave equations based on Euler instead Navier-Stokes but had in common the usage of high-order schemes in their simulations. However, recent works in CAA have used low-order schemes to discretise the convective term of Navier-Stokes equations while obtaining acceptable results. Tyacke et al. [16] used the second order Kinetic Energy Preserving of Jameson [17] in the simulation of a jet. Fuchs simulated a single stream jet [18] and the Rudimentary Landing Gear (RLG) [19] using a second-order scheme and obtaining very good acoustic results in both simulations.

Another aspect which has to be taken into account, apart from the order of the numerical scheme, is the non-linearities that mesh introduces; this happens when non-uniform uniform meshes are used. None of the previous commented authors in CAA used uniform meshes in their simulations but stretched ones. Vichnevetsky [20] or Trefethen [21], among others, have studied how mesh irregularities, such as a non constant spacing, are the source of additional errors in simulations.

Thus, the use of high-order schemes in wave-propagation simulations is still not enough justified. Extrapolating the conclusions extracted in the conditions of the classical approach to compute dispersion, i.e. uniform structured me
could not be the best recommended course of
ogy that avoids the linitations of the preyious
with any kind of numerical scheme.
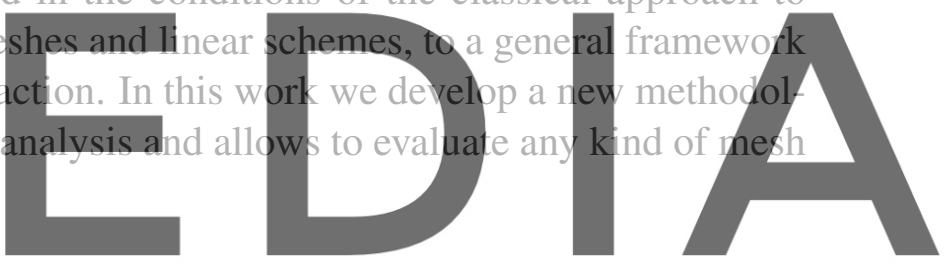

2 METHODOLOGY

Register for free at https//www.scipedia.com to download the version without the watermark 2.1 Analytical background

The Fourier Transform of a function $f(x): \mathbb{R} \mapsto \mathbb{R}$ is given by:

$$
\widehat{f}=\int_{-\infty}^{\infty} f(x) e^{-2 \pi i x \alpha} d x
$$

The Fourier Transform of the spatial derivative of $f(x)$ is:

$$
\widehat{f}^{\prime}(\alpha)=i \alpha \widehat{f}
$$

Reordering the last equation, is easy to isolate the analytical wavenumber as a function of $f$ and its derivative:

$$
\alpha=-i \frac{\widehat{f}^{\prime}(\alpha)}{\widehat{f}} .
$$


Nevertheless, when the space is discretised, previous equation does not hold. Applying both derivative and shifting theorems as Tam [7, 22], the discrete equivalent of Eq.(3) is obtained:

$$
\widetilde{\alpha}=\frac{-i}{\Delta x}\left[\sum_{k} a_{k} e^{i k \alpha \Delta x}\right] \text {. }
$$

As can be seen, previous definition implies a uniform spacing as a single $\Delta x$ appears. Thus, as the method requires using Fourier Transform properties, this analysis is restricted only to uniform meshes. However, we can see the Fourier Transform as a projection. Using the idea to project the function onto a set of orthonormal functions, we can avoid the limitations that the classical method has.

First, let's define the inner product of $\{\xi, \psi\} \in \mathcal{L}^{2}\left(\Omega_{\mu}, \mu\right)$, where $\mathcal{L}^{2}\left(\Omega_{\mu}, \mu\right)$ is the space of square Lebesgue-integrable functions $\psi: \Omega_{\mu} \subset \mathbb{R} \mapsto \mathbb{C}$ as:

$$
\langle\xi \mid \psi\rangle_{\Omega_{\mu}}=\int_{\Omega_{\mu}} \xi(\mu) \bar{\psi}(\mu) d \mu
$$

\section{Then, let $\Phi=\left\{\phi_{-N}(x), \phi_{-N+1}(x), \ldots \phi_{-1}(x), \phi_{0}(x), \phi_{1}(x), \phi_{2}(x), \ldots \phi_{N}(x)\right\}$ be an orthonormal} basis of functions of $\Omega_{x} \subset \mathbb{R}$
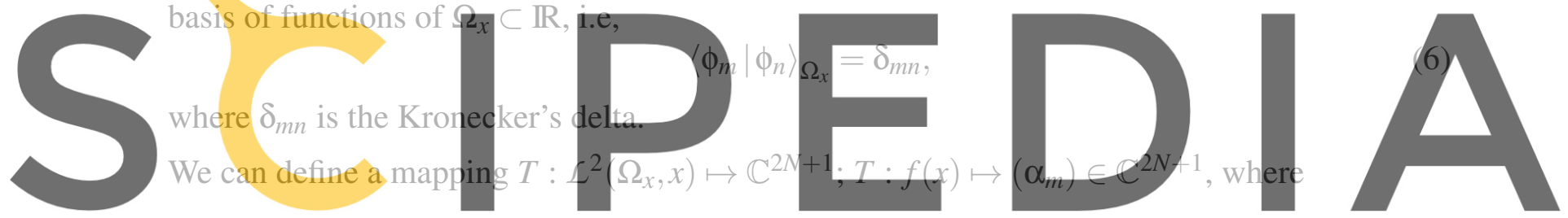

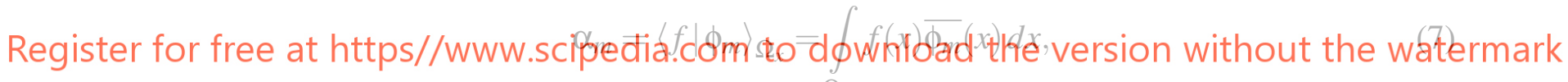

is the projection of $f(x)$ onto the $m$ function $\Phi$. Under the pertinent smoothness of $f$ criterion,

$$
f(x) \simeq S_{N}=\sum_{m=-N}^{N} \alpha_{m} \phi_{m}(x) ; \quad \lim _{N \rightarrow \infty} S_{N}=f(x) .
$$

This defines the inverse mapping $T^{-1}: \mathbb{C}^{2 N+1} \mapsto \mathcal{L}^{2}\left(\Omega_{x}, x\right), T:\left(\alpha_{m}\right) \in \mathbb{C}^{2 N+1} \mapsto f(x)$.

The derivative $f^{\prime}=\frac{d}{d x} f(x)$ can also be expressed in terms of its projection on the set of functions of $\Phi$ :

$$
f^{\prime}(x) \simeq S_{N}^{\prime}=\sum_{m=-N}^{N} \alpha_{m} \phi_{m}^{\prime}(x) \simeq \sum_{m=-N}^{N}\left(\alpha_{m} \sum_{n=-N}^{N} \gamma_{m n} \phi_{n}(x)\right)
$$

where

$$
\gamma_{m n}=\left\langle\phi_{m}^{\prime} \mid \phi_{n}\right\rangle_{\Omega_{x}}
$$


We define the matrix $\Gamma$, where its elements $(\Gamma)_{m n}=\gamma_{m n}$ represent the projections of the derivatives of $\phi_{m}$ with respect to " $x$ " on $\phi_{n}$.

$\Gamma$ characteristics are determined by the selected basis of functions $\Phi$ and the properties of the derivative operator. Assuming null contributions from boundaries and integrating by parts, it is straightforward to show that the derivative operator is skew-Hermitian with respect to the inner product of Eq.(5), i.e.,

$$
\left\langle\frac{d}{d x} \xi \mid \psi\right\rangle_{\Omega_{x}}=-\left\langle\xi \mid \frac{d}{d x} \psi\right\rangle_{\Omega_{x}} .
$$

Thus, $\Gamma$ is skew-Hermitian too.

Using an approximate derivative operator $\frac{\widetilde{d}}{d x}(\cdot)$, one gets $\widetilde{\Gamma} \in \mathbb{C}^{2 N+1 \times 2 N+1}$ instead of $\Gamma$. The errors associated with using approximate differential operators to approximate derivatives are the deviations of $\widetilde{\gamma}_{m n}$ from $\gamma_{m n}$. Finally, to ease the analysis, $\Phi$ can be chosen such that $\Gamma$ is diagonal and known, i.e., $\phi_{m}$ is an eigenfunction of the derivative. Then, the possible errors in $\widetilde{\Gamma}$ are:

- $\widetilde{\gamma}_{m n} \neq 0$ if $m \neq n$,

- $\operatorname{Re}\left(\widetilde{\gamma}_{m m}\right) \neq 0$, and $\frac{\operatorname{Im}\left(\widetilde{\gamma}_{m m}\right)}{\lambda_{m}} \neq 1$

where $\lambda_{m} \in \mathbb{I}$ is the eigenvalue or

2.2 Numerical method
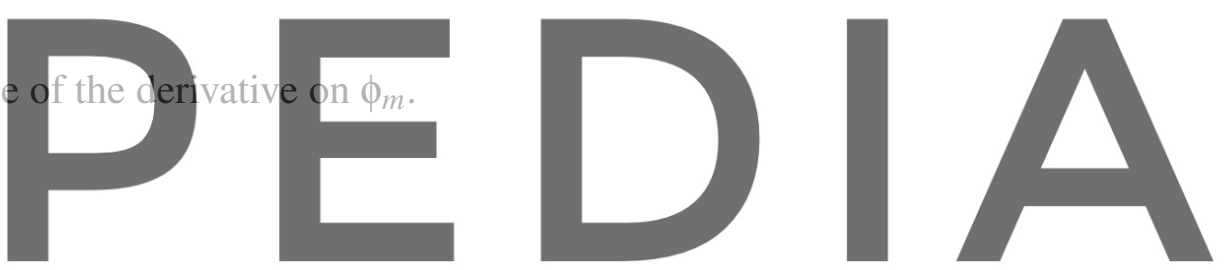

Defining the discrete differential operator as $A$, and splitting it into the Hermitian, $D$, and skew-

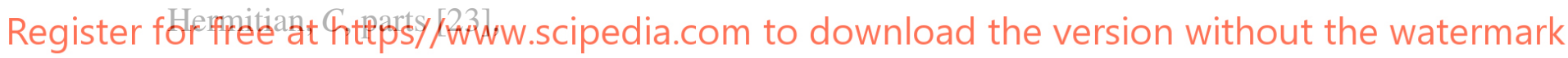

$$
\begin{aligned}
& C=\frac{1}{2}\left(A-A^{*}\right) ; \\
& D=\frac{1}{2}\left(A+A^{*}\right) ; \\
& A=C+D,
\end{aligned}
$$

where $(\cdot)^{*}$ indicates the conjugate transpose. The matrices $C$ and $D$ that appear after splitting the discrete differential operator have interesting properties involving the inner product:

$$
\begin{gathered}
\langle C \psi \mid \psi\rangle \in \mathbb{I} \\
\langle D \psi \mid \psi\rangle \in \mathbb{R} \\
\langle C \psi \mid \eta\rangle=-\langle\psi \mid C \eta\rangle \\
\langle D \psi \mid \eta\rangle=\langle\psi \mid D \eta\rangle,
\end{gathered}
$$

where $\{\psi, \eta\} \in \mathbb{C}^{N}$. 
This matrices and $\Gamma$ are closely related. More precisely, matrix $C$ is related to the real part of the scalars $\gamma$ that have been defined on Eq.(10). $D$ is related to the imaginary part of these values. When a discretization is used, i.e. a matrix $A$ is defined, these scalars can be numerically calculated:

$$
\begin{gathered}
\widetilde{\gamma}_{m n}=\left\langle A \phi_{m} \mid \phi_{n}\right\rangle \\
\widetilde{\gamma}_{m n}^{C}=\operatorname{Im}\left(\widetilde{\gamma}_{m n}\right)=\left\langle C \phi_{m} \mid \phi_{n}\right\rangle \\
\widetilde{\gamma}_{m n}^{D}=\operatorname{Re}\left(\widetilde{\gamma}_{m n}\right)=\left\langle D \phi_{m} \mid \phi_{n}\right\rangle .
\end{gathered}
$$

This development holds because $\Phi$ is orthonormal and due to the nature of the skew and Hermitian operators. They can be calculated from $A$ :

$$
\begin{gathered}
\widetilde{\gamma}_{m n}^{C}=\left\langle C \phi_{m} \mid \phi_{n}\right\rangle=\frac{\left\langle A \phi_{m} \mid \phi_{n}\right\rangle-\left\langle\phi_{m} \mid A \phi_{n}\right\rangle}{2} \\
\widetilde{\gamma}_{m n}^{D}=\left\langle D \phi_{m} \mid \phi_{n}\right\rangle=\frac{\left\langle A \phi_{m} \mid \phi_{n}\right\rangle+\left\langle\phi_{m} \mid A \phi_{n}\right\rangle}{2},
\end{gathered}
$$

where the values of $\widetilde{\gamma_{m n}}$ are the different elements of the matrix $\widetilde{\Gamma}$. To simplify the analysis, the root mean square of the second index of the values of $\widetilde{\gamma}$ can be calculated,

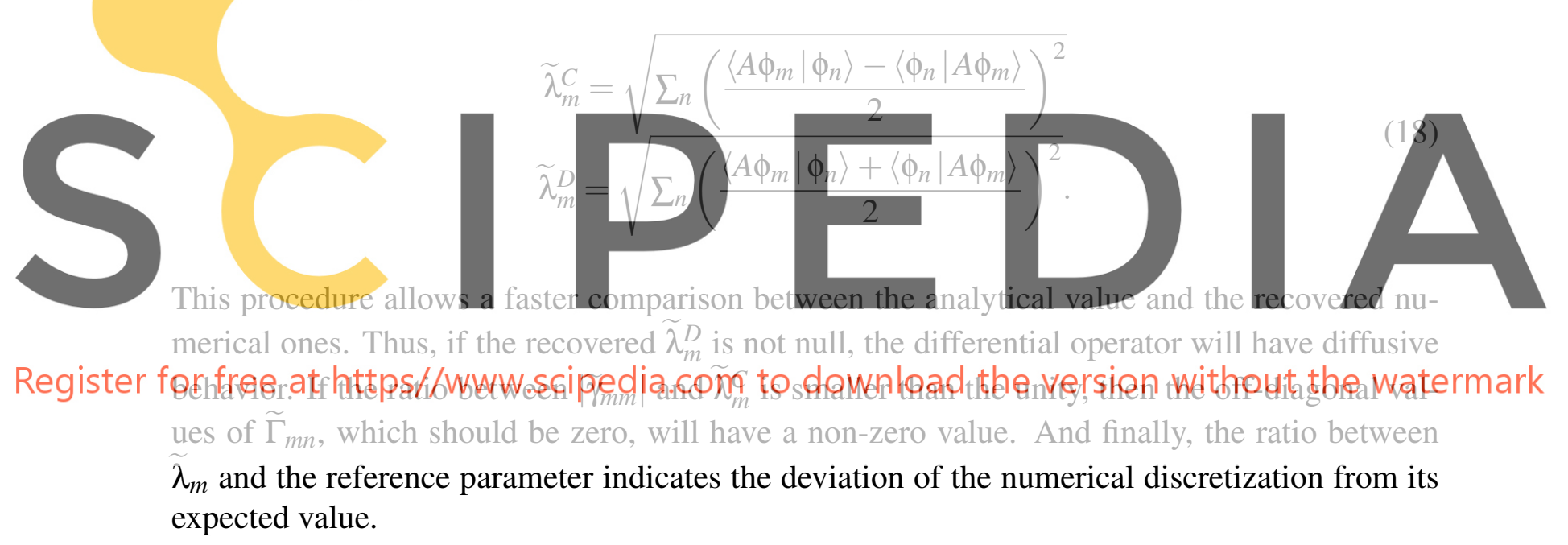

\subsubsection{Orthonormal basis}

Once the method is defined, the next step is choosing an appropriate orthonormal basis. Here we propose to use the eigenvectors of the discrete Laplacian operator. The main reasons can be sumarized as:

- They retain the concept of mesh connectivity as they are calculated from the discrete Laplacian, which takes into account how mesh nodes are linked between them.

- In evenly spaced domains, the eigenvectors of the discrete Laplacian are, indeed, discrete sinusoids. 
- And finally, in the continuous limit, or as we refine the mesh, the eigenvectors and eigenvalues of the discrete Laplacian collapse onto the eigenfunctions and eigenvectors of the continuous Laplacian, i.e. sinusoids.

Additionally, the discrete Laplacian $L$ and the dicrete gradient $G$ are related [24]:

$$
L=G^{*} G
$$

The Singular Value Decomposition of $\mathrm{G}$ relates its right eigenvectors and singular values with the right eigenvectors and eigenvalues of L. More precisely, G and L share the same eigenvectors and the eigenvalues of $\mathrm{G}$ are the square-root of the eigenvalues of $\mathrm{L}$.

\subsubsection{Phase effect}

When non-linear schemes or non-uniform meshes are used, the derivative is distorted. More precisely, the derivation process projects each eigenmode onto several components instead a single one. In uniform meshes and using non-linear discrete schemes, Fauconnier and Dick [10] tackled this problem by setting the phase inside the sinusoids in the Fourier Transform as a random parameter; their idea was that the numerical wavenumber is a function of both analytical wavenumber and the random phase. Repeating the process 5000 times, they finally computed an averaged numerical wavenumber which did not depend on the phase.

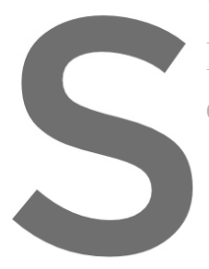

In our case this is equiva conforms the base, with the ro
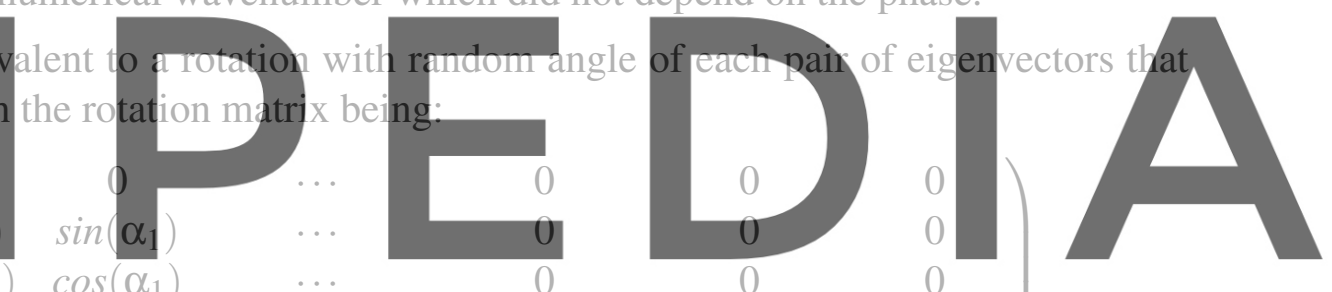

Register for

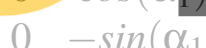

$\cos \left(\alpha_{1}\right)$

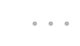

in to

$R=\mid \begin{array}{cc}0 & 0 \\ \vdots & \\ 0 & 0 \\ 0 & 0 \\ 0 & 0 \\ 0 & 0\end{array}$

The eigenvectors in the rotated base will be:

$$
E^{\prime}=R E,
$$

where $E^{\prime}$ are the eigenvectors in the rotated basis and $E$ the eigenvectors in the original one.

Differently than with uniform meshes, where each Laplacian eigenvalue appears twice (except 0 and the maximum one for meshes with even number of nodes), non-uniform meshes does not have this property. This means that an effective eigenvalue is computed: the average between two consecutive eigenvalues that are associated with the two rotated eigenvectors. 


\begin{tabular}{cc|cc} 
Acronym & Convective scheme & Acronym & Convective scheme \\
\hline UPW & First-order upwind & MM & Minmod flux-limiter \\
SP2 & Second-order symmetric & SB & Superbee flux-limiter \\
DRP4 & Fourth-order DRP & VL & Van Leer flux-limiter \\
DRP6 & Sixth-order DRP & WENO3 & Third-order WENO \\
SP6 & Sixth-order symmetric & WENO5 & Fifth-order WENO \\
MLS3 & Sixth-order Moving Least-Squares & WENO7 & Seventh-order WENO \\
\hline
\end{tabular}

Table 1: Acronyms of the convective schemes.

\section{NUMERICAL TESTS AND RESULTS}

Even though the procedure developed here could be used in any kind of mesh, such as 3D Cartesian or unstructured, it is important to take into account two critical facts:

- First, structured meshes are the most common approach in the CAA community. So, it would make sense to study the same kind of meshes that are used by the majority. Additionally, working with fully unstructured meshes implies that the recovered eigenvalue cannot be decomposed onto its Cartesian components. In other words, we will recover
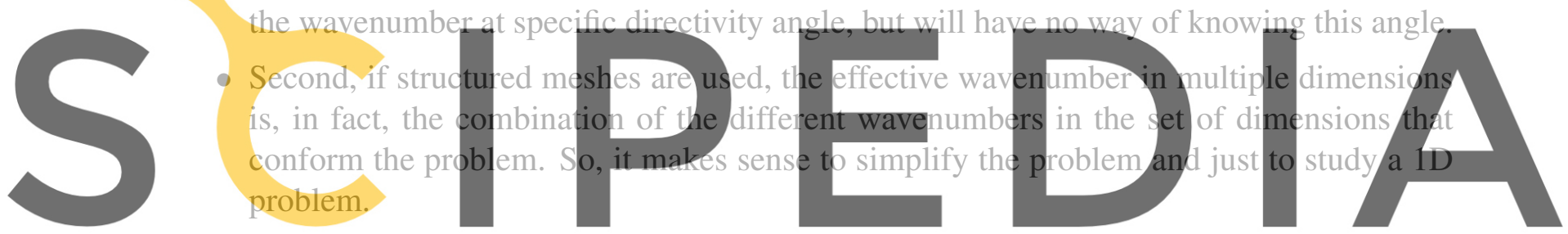

Consequently, the meshes used in this article are just structured one-dimensional meshes. How-

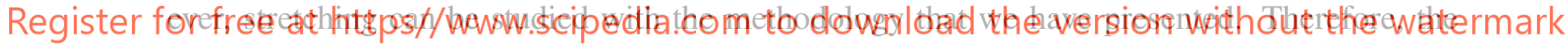
mesh set will include both uniform and non-uniform meshes. The mesh characteristics can be summarized as:

- 6 different Poisson ratios: from 0 to $5 \%$.

- 5 different minimum mesh sizes: from $1 / 32$ to $1 / 512$.

Regarding the convective schemes, several of both linear and non-linear ones will be studied. For the sake of clarity, in Table 1 we include the acronyms of the different convective schemes that have been studied. However, we do not include the legend of meshes; the reasons will be commented with the results.

\subsection{Dispersion error results}

In Figures 1 and 2 we have included the recovered eigenvalue, using Eq.(18) and the concept of basis rotation, for several schemes; then, this value is multiplied by the maximum mesh size. 

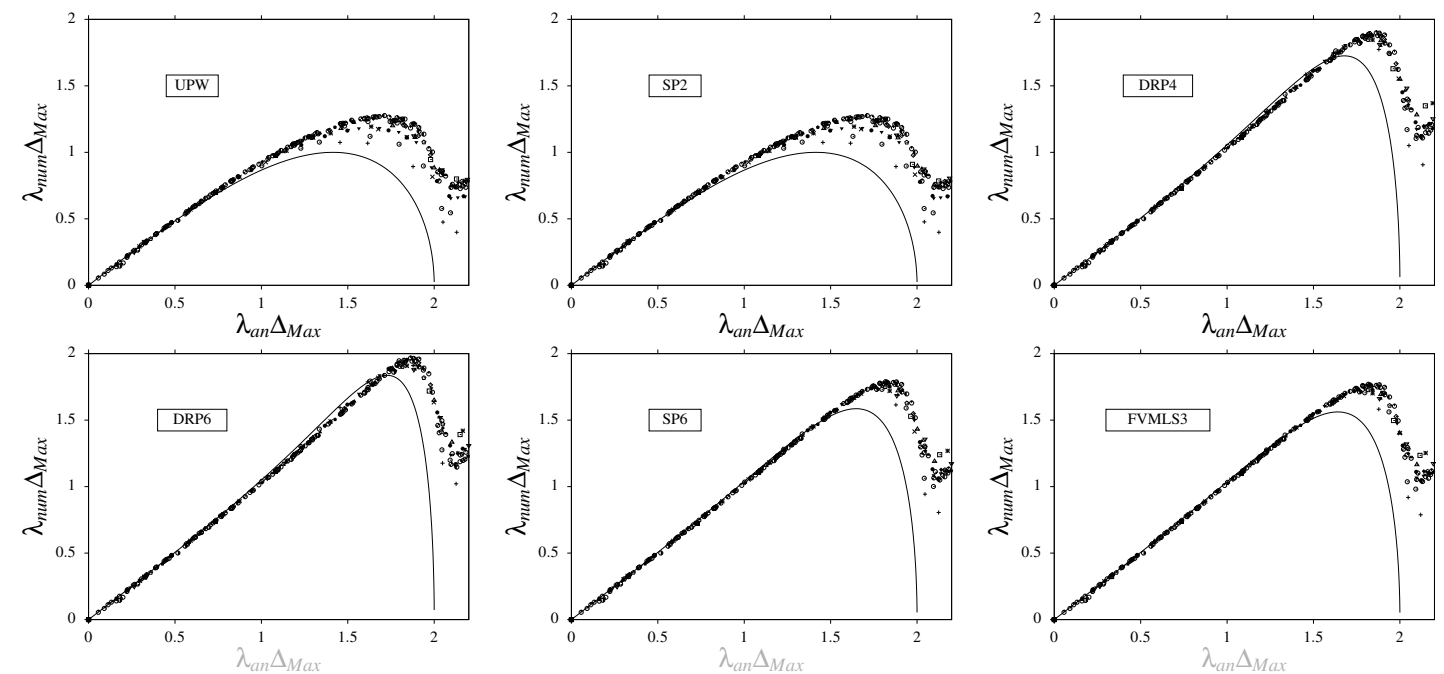

Figure 1: Recovered vs analytical eigenvalue made non-dimensional via maximum mesh size. Linear schemes.
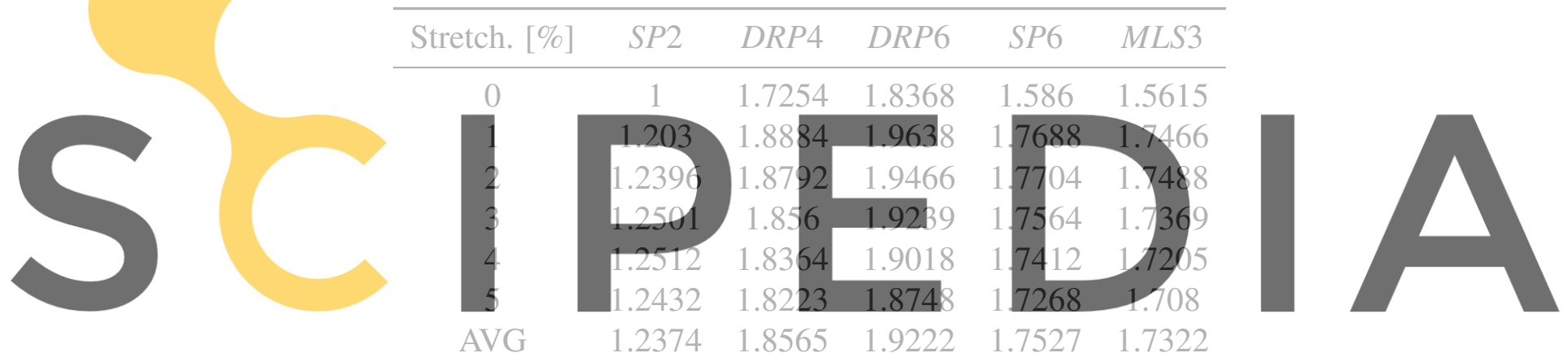

Register for free at https//WwW.scipedia, com to download the version without the watermark symmetry preserving in uniform meshes, linear schemes.

As can be seen in these figures, only two kind of plots are observed: in solid line, results corresponding to uniform meshing; the dots, belong to stretched meshes. Data show that stretched meshes collapse onto the same plot, independently of the stretching factor used. There is, indeed, a fast transition from the uniform plot towards the stretched one.

In Tables 2 and 3 we have included the evolution of the peak in these plots, which is a signal from the transition from the uniform to the stretched plot. Low-order schemes exhibit a slow transition from uniform plot towards the stretched one, as the maximum peak is achieved for highly stretched meshes. On the other hand, high-order schemes almost do not have this transition; for slightly stretched meshes they achieve the maximum peak and the kind of plot typical for stretched meshes. Therefore, it seems that high-order schemes are more sensitive to mesh transformations, as they behave more differently faster than low-order schemes. 

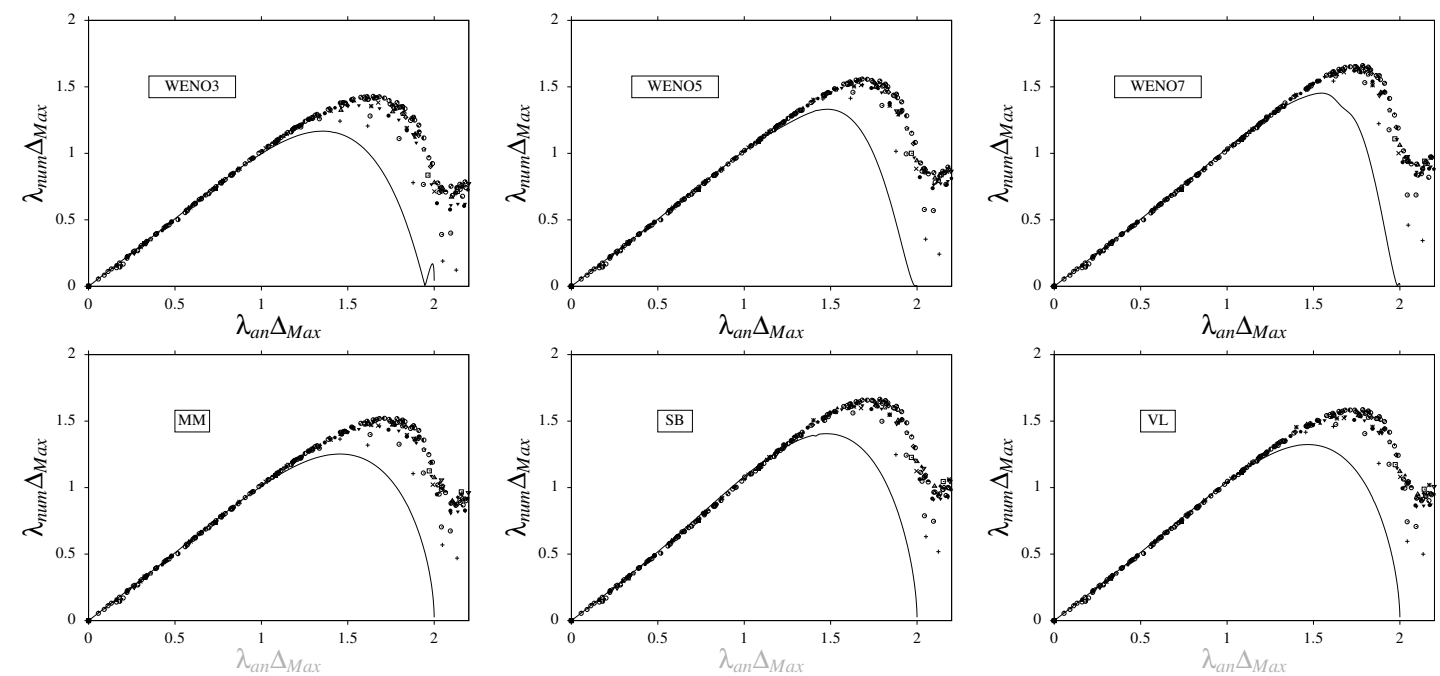

Figure 2: Recovered vs analytical eigenvalue made non-dimensional via maximum mesh size. Non-linear schemes.

And finally, all stretched plots share the same common feature: around $\lambda \Delta_{\operatorname{Max}} \sim 2$ there is a sudden drop of the recovered value; this is an indicative of the maximum allowed mode in the mesh, which just depends on the maximum mesh size. And this happens just with the eigenval-

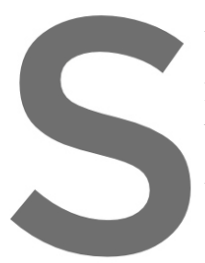
ues, not the frequencies.

for all the studied meshe

but a lot of them. Consequently, there is not

the maximum mesh size
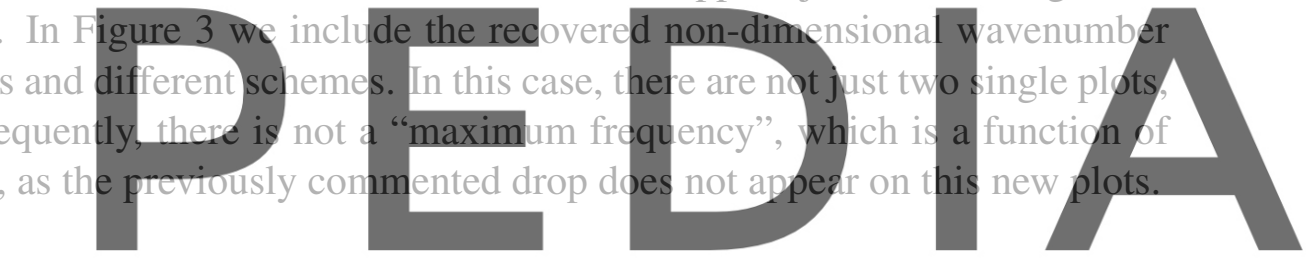

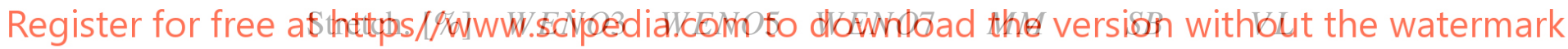

\begin{tabular}{ccccccc}
0 & 1.1667 & 1.3317 & 1.4529 & 1.2526 & 1.4053 & 1.3237 \\
1 & 1.3747 & 1.52 & 1.6315 & 1.4713 & 1.6272 & 1.543 \\
2 & 1.4036 & 1.5402 & 1.6432 & 1.4946 & 1.6444 & 1.566 \\
3 & 1.4072 & 1.5362 & 1.6377 & 1.4966 & 1.637 & 1.5611 \\
4 & 1.4047 & 1.5296 & 1.6249 & 1.4973 & 1.6344 & 1.5573 \\
5 & 1.3889 & 1.5183 & 1.6132 & 1.4736 & 1.6205 & 1.5374 \\
AVG & 1.3958 & 1.5289 & 1.6301 & 1.4867 & 1.6327 & 1.553 \\
\hline
\end{tabular}

Table 3: Non-dimensional maximum eigenvalue normalized respect to the maximum eigenvalue for first-order upwind in uniform meshes, non-linear schemes. 

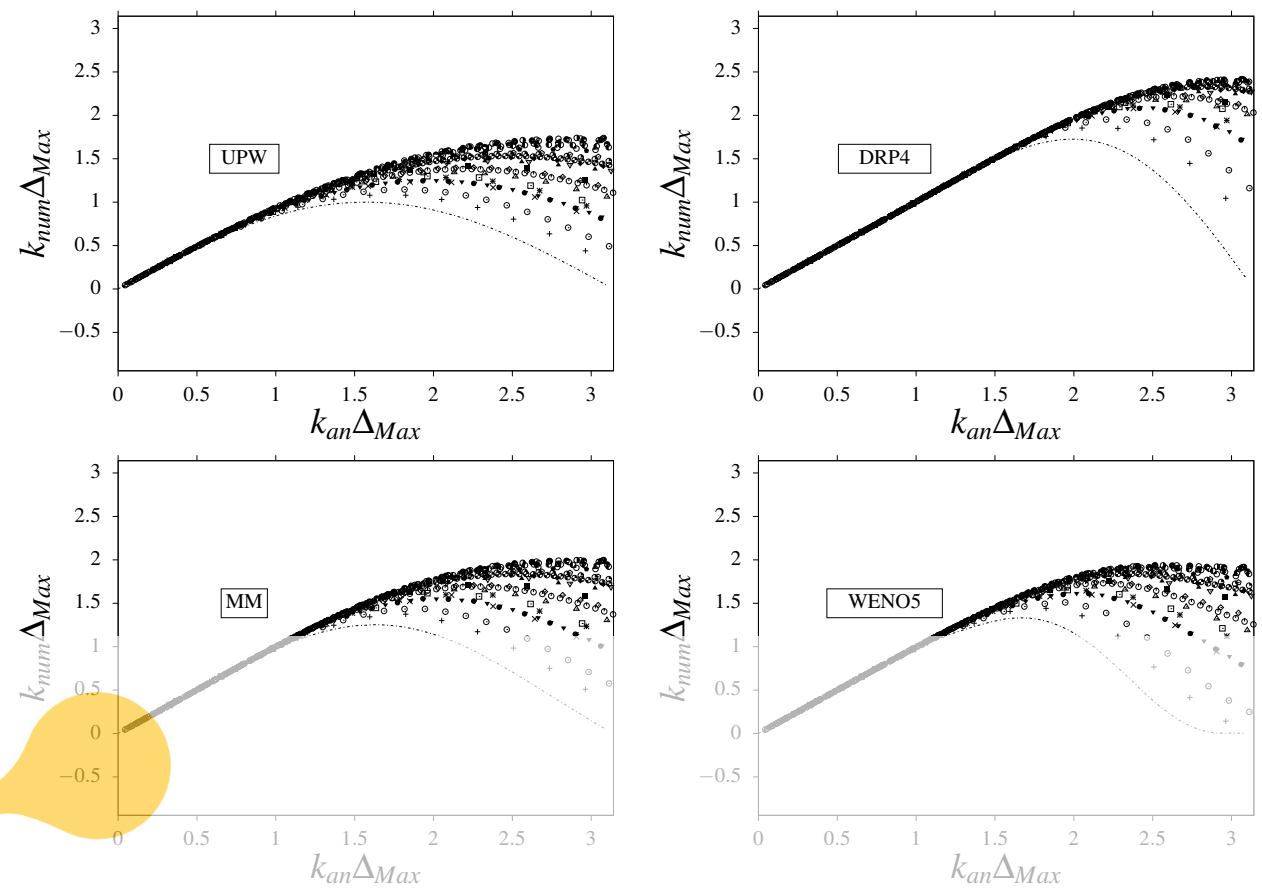

Figure 3: Recovered vs analytical wavenumber made non-dimensional via maximum mesh size.
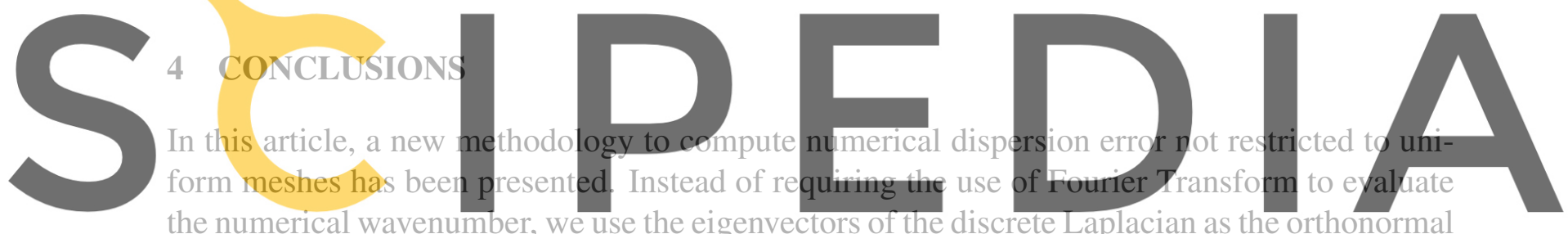

the numerical wavenumber, we use the eigenvectors of the discrete Laplacian as the orthonormal

Register for free at https//www.scipedia.com to download the version without the watermark Non-dimensional results of the recovered eigenvalue via the maximum mesh size show that two different plots are recovered: one for uniform meshing and other for stretched meshes, independently of the studied stretching factor the plots obtained collapse onto a single one.

In the studied plots for stretched meshes, a sudden drop in the recovered eigenvalue, i.e. an increase of the numerical error, always appears. This drop is always located around $\lambda \Delta_{\text {Max }} \sim$ 2. However, recovered non-dimensional wavenumber, via base change, do not exhibit this drop. Moreover, the plots for different meshes are a function of both maximum mesh size and used stretching factor, as the results do not collapse onto a single plot. This reforces the idea that stretched meshes have a maximum allowed eigenvalue rather than a maximum allowed frequency.

As a further work, we would like that the herepresented methodology could help to select the most appropriate scheme for a given mesh geometry or be used to design meshing techniques leading to a reduction in the dispersion error. 


\section{Acknowledgements}

This work has been financially supported by the Ministerio de Economía y Competitividad, Spain (No. ENE2017-88697-R). J.R.P. is supported by a FI-DGR 2015 predoctoral contract financed by Generalitat de Catalunya, Spain.

\section{REFERENCES}

[1] F. X. Trias, O. Lehmkuhl, A. Oliva, C. D. Pérez-Segarra, and R. Verstappen, "Symmetrypreserving discretization of navier-stokes equations on collocated unstructured grids," Journal of Computational Physics, vol. 258, pp. 246-267, 2014.

[2] R. Verstappen and A. Veldman, "Symmetry-preserving discretization of turbulent flow," Journal of Computational Physics, vol. 187, no. 1, pp. 343-368, 2003.

[3] W. Rozema, J. Kok, R. Verstappen, and A. Veldman, "A symmetry-preserving discretisation and regularisation model for compressible flow with application to turbulent channel flow," Journal of Turbulence, vol. 15, no. 6, pp. 386-410, 2014.

[4] X.-D. Liu, S. Osher, and T. Chan, "Weighted essentially non-oscillatory schemes," Journal of Computational Physics, vol. 115, no. 1, pp. 200-212, 1994.

[5] A. Harten, B. Engquist, S. Osher, and S. R. Chakravarthy, "Uniformly high order accurate essentially non-oscillatory schemes, iii," in Upwind and high-resolution schemes, pp. 218290, Springer, 1987.

[6] B. Van Leer, "Towards the ultimate conservative difference scheme. ii. monotonicity and conservation combined in a second-order scheme," Journal of Computational Physics, vol. 14, no. 4, pp. 361-370, 1974.

[7] C. K. W. Tam and J. C. Webb, "Dispersion-relation-preserving finite difference schemes for Computational acoustics," Journal of Computational Physics, pp. 262-281, 1993.

[8] S. K. Lele, "Compact finite difference schemes with spectral-like resolution," Journal of Computational Physics, 1992.

[9] S. Pirozzoli, "On the spectral properties of shock-capturing schemes," Journal of Computational Physics, vol. 219, no. 2, pp. 489-497, 2006.

[10] D. Fauconnier and E. Dick, "On the spectral and conservation properties of nonlinear discretization operators," Journal of Computational Physics, vol. 230, no. 12, pp. 44884518, 2011.

[11] C. Bogey, "Grid sensitivity of flow field and noise of high-reynolds-number jets computed by large-eddy simulation," International Journal of Aeroacoustics, vol. 17, no. 4-5, pp. 399-424, 2018.

[12] M. L. Shur, P. R. Spalart, and M. K. Strelets, "Noise prediction for increasingly complex 
jets. part i: Methods and tests," International Journal of Aeroacoustics, vol. 4, no. 3, pp. 213-245, 2005.

[13] M. L. Shur, P. R. Spalart, and M. K. Strelets, "Jet noise computation based on enhanced des formulations accelerating the rans-to-les transition in free shear layers," International Journal of Aeroacoustics, vol. 15, no. 6-7, pp. 595-613, 2016.

[14] J. H. Seo and Y. J. Moon, "Linearized perturbed compressible equations for low mach number aeroacoustics," Journal of Computational Physics, vol. 218, no. 2, pp. 702-719, 2006.

[15] R. Ewert and W. Schröder, "Acoustic perturbation equations based on flow decomposition via source filtering," Journal of Computational Physics, vol. 188, no. 2, pp. 365-398, 2003.

[16] J. Tyacke, I. Naqavi, Z.-N. Wang, P. Tucker, and P. Boehning, "Predictive large eddy simulation for jet aeroacoustics-current approach and industrial application," Journal of Turbomachinery, vol. 139, no. 8, p. 081003, 2017.

[17] A. Jameson, "Formulation of kinetic energy preserving conservative schemes for gas dynamics and direct numerical simulation of one-dimensional viscous compressible flow in a shock tube using entropy and kinetic energy preserving schemes," Journal of Scientific Computing, vol. 34, no. 2, pp. 188-208, 2008.

[18] M. Fuchs, C. Mockett, M. Shur, M. Strelets, and J. Kok, "Single-stream round jet at m= 0.9," in Go4Hybrid: Grey Area Mitigation for Hybrid RANS-LES Methods, pp. 125-137, Springer, 2018.

[19] M. Fuchs, C. Mockett, F. Kramer, T. Knacke, D. Fischer, J. Sesterhenn, and F. Thiele, "Assessment of different meshing strategies for low mach number noise prediction of a rudimentary landing gear," in 23rd AIAA/CEAS Aeroacoustics Conference, p. 3020, 2017.

[20] R. Vichnevetsky, "Propagation through numerical mesh refinement for hyperbolic equations," Mathematics and Computers in Simulation, vol. 23, no. 4, pp. 344-353, 1981.

[21] L. N. Trefethen, "Group velocity in finite difference schemes," SIAM review, vol. 24, no. 2, pp. 113-136, 1982.

[22] C. K. Tam, Computational Aeroacoustics: a wave number approach, vol. 33. Cambridge University Press, 2012.

[23] L. Beilina, E. Karchevskii, and M. Karchevskii, Numerical Linear Algebra: Theory and Applications. Springer, 2017.

[24] P. B. Bochev and J. M. Hyman, Principles of mimetic discretizations of differential operators. Springer, 2006. 